\title{
Susceptibility of XPD and hOGG1 genetic variants to prostate cancer
}

\author{
CHENG ZHOU $^{1}$, LI-PING XIE ${ }^{2}$, YI-WEI LIN ${ }^{2}$, KAI YANG $^{2}$, QI-QI MAO ${ }^{2}$ and YUE CHENG ${ }^{1}$ \\ ${ }^{1}$ Department of Urology, Ningbo First Hospital, Ningbo, Zhejiang 315000; ${ }^{2}$ Department of Urology, \\ First Affiliated Hospital, Zhejiang University School of Medicine, Hangzhou, Zhejiang 310003, P.R. China
}

Received March 27, 2013; Accepted May 24, 2013

DOI: $10.3892 /$ br.2013.123

\begin{abstract}
DNA repair genes are important in maintaining genomic stability and integrity. DNA repair gene polymorphisms, such as those of the human homolog of 8-oxoguanine DNA glycosylase 1 (hOGG1) and excision repair cross-complementing rodent repair deficiency, complementation group 2/Xeroderma pigmentosum complementation group D (ERCC2/XPD), contribute to carcinogenesis. The aim of this study was to investigate the association of prostate cancer (PCa) risk with hOGG1 and ERCC2/XPD genetic variants. A case-control study of 200 cases including $100 \mathrm{PCa}$ patients and 100 healthy subjects was conducted. Two single-nucleotide polymorphisms (SNPs) (ERCC2/XPD Arg156Arg and hOGG1 Ser326Cys) were genotyped by polymerase chain reaction-restriction fragment length polymorphism (PCR-RFLP). The results demonstrated a significant association of the XPD156 homozygous (AA, OR=3.80; 95\% CI: 1.19-12.18; $\mathrm{P}=0.017$ ), heterozygous $(\mathrm{AC}, \mathrm{OR}=2.48 ; 95 \% \mathrm{CI}: 1.02-6.35$; $\mathrm{P}=0.033)$ and combined (AA+AC, $\mathrm{OR}=2.76 ; 95 \% \mathrm{CI}: 1.18-6.84$; $\mathrm{P}=0.011)$ mutant genotypes with a predisposition to high-risk $\mathrm{PCa}$. In the stratified analysis, predisposition to high-risk $\mathrm{PCa}$ was also associated with the mutant genotypes of hOGG1 326 homozygous mutant (GG, OR=2.93; 95\% CI: 1-8.74; $\mathrm{P}=0.033$ ). The results also showed that the A allele of XPD Arg156Arg and the $\mathrm{G}$ allele of hOGG1 Ser326Cys were associated with an increased risk of $\mathrm{PCa}(\mathrm{OR}=1.86$ and $1.62 ; 95 \% \mathrm{CI}: 1.13-3.06$ and 1-2.67, respectively). In conclusion, the findings of this study demonstrated that the ERCC2/XPD Arg156Arg and hOGG1 Ser326Cys polymorphisms increased the susceptibility to high-risk PCa.
\end{abstract}

Correspondence to: Dr Cheng Zhou, Department of Urology, Ningbo First Hospital, Liu Ting Street, Ningbo, Zhejiang 315000, P.R. China

E-mail: zhoucheng2006zc@126.com

Key words: prostate cancer, single-nucleotide polymorphism, excision repair cross-complementing rodent repair deficiency, complementation group 2/Xeroderma pigmentosum complementation group D, human homolog of the 8-oxoguanine DNA glycosylase 1

\section{Introduction}

Prostate cancer ( $\mathrm{PCa})$ is among the most common malignant tumors among Western men, ranking second only to lung cancer regarding cancer-related mortality (1). The incidence of $\mathrm{PCa}$ among Asian men is significantly lower; however, the incidence of PCa in China has increased significantly over the last few years (2). The etiology of PCa has not been fully elucidated. It is a multifactorial disease, with genetic and environmental factors contributing to its incidence. In addition, several risk factors, such as ethnicity, family history and age, have been associated with an increased PCa risk $(3,4)$. The exponential increase in the risk of $\mathrm{PCa}$ associated with aging may reflect the accumulation of DNA damage resulting from a series of processes, such as oxidative stress, inflammation and environmental carcinogens, or a decrease in the DNA damage-repair response capacity. DNA repair is essential to the ability of response to environmental carcinogen-induced damage. DNA repair mechanisms are important pathways in the removal of oxidative DNA compounds or DNA adducts from damaged genomic sites (5). The key DNA repair pathways that are often associated with cancer risk are listed as follows: The base excision repair (BER) removes simple base modifications, such as single-strand breaks, oxidative DNA damage and alkylation and non-bulky adducts (6). The nucleotide excision repair (NER) removes larger lesions, which often result from environmental damage, such as UV radiation and external carcinogens (7). The mismatch repair is considered to involve MLH1, MSH2, PMS2 and MSH6 in damage recognition, followed by excision, polymerization and ligation. The double-strand-break repair consists of the homologous recombination and the non-homologous end joining pathways. Among these pathways, NER and BER are the most significant (8).

In this study, we investigated the polymorphisms of the excision repair cross-complementing rodent repair deficiency, complementation group 2/Xeroderma pigmentosum complementation group D (ERCC2/XPD) and the human homolog of the 8-oxoguanine DNA glycosylase 1 (hOGG1) genes. ERCC2/XPD, a gene involved in NER and basal transcription, may influence individual DNA repair capacity, particularly of bulky adducts (9). A previous epidemiological study (10) evaluated the potential role of the ERCC2 Arg156Arg polymorphism (rs238406) in cancer; however, cross-study results have been conflicting. 
hOGG1 is a protein involved in the BER pathway, which is responsible for repairing one of the most mutagenic lesions among base modifications, 8-hydroxyguanine (8-oxoG). Previous epidemiological studies (11-13) investigated the association between the Ser326Cys polymorphism (rs1052133) in the hOGG1 gene and the risk for different types of cancer. Significant increases were observed in the risk of esophageal (11), lung (12) and colon cancer (13) in association with the hOGG1 326 polymorphism.

A previous study by Song et al (14) investigated the trend of PCa incidence in the urban area of Tianjin, China, between 1981 and 2004, to provide a scientific rationale for the prevention and control of PCa. The authors concluded that, despite the currently low incidence of $\mathrm{PCa}$ in Tianjin, $\mathrm{PCa}$ is increasing rapidly. Consequently, we investigated the association of $\mathrm{PCa}$ occurrence and progression with the variants of the ERCC2/XPD and hOGG1 genes in a southeastern Chinese population. The codon 156 (Arg156Arg) polymorphism in XPD and the codon 326 (Ser326Cys) polymorphism in hOGG1 were investigated.

\section{Materials and methods}

Study population. A total of 100 patients with pathologically confirmed PCa and 100 age-matched control individuals were enrolled in this study (Table I). The mean age of the patient and control groups was $69.99 \pm 8.59$ and $66.91 \pm 11.89$ years, respectively. The 100 patients and the 100 cancer-free control subjects were enrolled at the First Affiliated Hospital, School of Medicine, Zhejiang University and the Ningbo First Hospital, between May, 2011 and October, 2012. The PCa patient age range was 46-90 years. All the cases were diagnosed within 1 year of enrollment. All the controls were non-cancer patients admitted to the other wards (day and cadre wards) in the same region, or were enrolled among individuals undergoing regular physical exams in the hospital. Randomly selected controls were age- and gender-matched to the cases $(\sim 1: 1)$. Written informed consent was obtained from all patients and the study was approved by the hospital boards and Ethics Committees.

Genotype. DNA was isolated from blood samples collected from PCa patients and control subjects using the QIAamp DNA Blood Mini kit (Qiagen Inc., Hilden, Germany) following the manufacturer's protocol, quantified using the GeneQuant ${ }^{\mathrm{TM}}$ pro spectrophotometer (Amersham Biosciences, Piscataway, NJ, USA) and stored at $-20^{\circ} \mathrm{C}$. The XPD Arg156Arg (rs238406) polymorphism was detected using a polymerase chain reactionrestriction fragment length polymorphism (PCR-RFLP)-based method (5). Each PCR was performed in a total volume of $20 \mu \mathrm{l}$, consisting of $0.3 \mu \mathrm{l}$ of a $10-\mu \mathrm{M}$ solution of each primer, $1.5 \mathrm{mM} \mathrm{MgCl}_{2}, 0.8 \mathrm{mM}$ dNTP, 0.5 units RedTaq DNA polymerase (Sigma, St. Louis, MO, USA), $1 \mu 1$ genomic DNA (80 ng/ $/ \mathrm{l}$ ) and $15.6 \mu \mathrm{l} \mathrm{H} \mathrm{H}_{2} \mathrm{O}$, using a PTC-200 Thermal Cycler (MJ Research Inc., Watertown, MA, USA). The thermal cycling conditions were as follows: an initial denaturation step at $95^{\circ} \mathrm{C}$ for $5 \mathrm{~min}, 30$ cycles at $95^{\circ} \mathrm{C}$ for $30 \mathrm{sec}$, annealing at $60^{\circ} \mathrm{C}$ for $30 \mathrm{sec}$ and extension at $72^{\circ} \mathrm{C}$ for $1 \mathrm{~min}$. The final extension was performed at $72^{\circ} \mathrm{C}$ for $7 \mathrm{~min}$. The PCR primers were synthesized by Takara Biotechnology Co., Ltd., (Dalian, China) and were as follows: forward, 5'-AGGGTTTGAAGAGTGGTTGG-3'
Table I. Patient and tumor characteristics.

\begin{tabular}{lcc}
\hline Characteristics & Cases $(\mathrm{n}=100)$ & Controls $(\mathrm{n}=100)$ \\
\hline Age $(\text { mean } \pm \mathrm{SD})^{\mathrm{a}}$ & $69.99 \pm 8.59$ & $66.91 \pm 11.89$ \\
PSA $(\mathrm{ng} / \mathrm{ml})$ & & \\
$<10$ & 25 & \\
$10-20$ & 35 & \\
$>20$ & 40 & \\
Gleason score & & \\
$\leq 6$ & 24 & \\
7 & 46 & \\
$\geq 8$ & 30 & \\
pT stage & \\
$\leq \mathrm{T} 2 \mathrm{a}$ & 54 \\
T2b & 33 \\
$\geq \mathrm{T} 2 \mathrm{c}$ & 13 \\
\hline
\end{tabular}

${ }^{\mathrm{a} C a s e s}$ vs. controls, $\mathrm{P}>0.05$. SD, standard deviation; PSA, prostate-specific antigen.

and reverse 5'-TCAGGTCATCCAGGTTGTAG-3'. The primers were digested with TfiI enzyme (New England Biolabs, Beverly, MA, USA). An aliquot of $10 \mu \mathrm{l}$ PCR product was digested with 3 units TfiI enzyme. The three possible genotypes were defined by three distinct banding patterns: CC (350 bp fragments), AC (350, 170 and $180 \mathrm{bp}$ fragments) and AA (170 and $180 \mathrm{bp}$ fragments) (Fig. 1). For hOGG1 Ser326Cys (rs1052133), the PCR products were amplified with the primers 5'-ACTGTCACTAGTCTCACCAG-3' (sense) and 5'-GGAAGGTGCTTGGGGAAT-3' (antisense), then digested with Fnu4HI (New England Biolabs). The PCR product was $200 \mathrm{bp}$ in length. It was digested by the Fnu4HI restriction enzyme into two 100-bp fragments for the 326Cys allele but could not be digested for the 326 Ser allele. Fragments were separated on a $2 \%$ agarose gel and stained with ethidium bromide. The Cys/Cys homozygote was cleaved by Fnu4HI and yielded a 100-bp band. The Ser/Ser homozygote could not be cleaved by Fnu4HI and remained a single 200-bp band. The Ser/Cys heterozygote contained both the 200- and 100-bp bands. The image of a representative gel is presented in Fig. 2. The results of RFLP-PCR were validated by DNA sequencing.

Statistical analysis. The demographic data of the study groups were compared by the Chi-square and Student's t-tests. Each polymorphism in the control group was tested for deviation from the Hardy-Weinberg equilibrium (HWE) by comparing the observed and expected genotype frequencies using the Chi-square test. Analyses were performed using SPSS software version 16.0 (SPSS, Inc., Chicago, IL, USA).

\section{Results}

Characteristics of PCa patients and control subjects. The mean age, serum prostate-specific antigen (PSA), Gleason score and pT stage of individual PCa patients are shown in Table I. The two-tailed Student's t-test was used to compare the distribution of age between patients and control subjects. 
Table II. Ser326Cys polymorphism of the hOGG1 gene and Arg156Arg polymorphism of the ERCC2/XPD gene in PCa patients.

\begin{tabular}{lcccc}
\hline $\begin{array}{l}\text { Polymorphism } \\
\text { genotype or allele }\end{array}$ & Patients $(\mathrm{n}=100)$ & OR & \\
\hline Ser326Cys & & & 1.00 & \\
CC & 22 & 29 & 1.20 & $(05 \%$ CI $)$ \\
CG & 52 & 57 & 2.45 & $(0.96-2.49)$ \\
GG & 26 & 14 & 1.45 & $(0.73-2.90)$ \\
GG+CG & 78 & 71 & 1.47 & $(0.97-2.22)$ \\
C & 104 & 85 & 1.00 & \\
G & 96 & 115 & 1.00 & \\
Arg156Arg & & & 1.58 & $(0.80-3.13)$ \\
CC & 26 & 38 & 2.36 & $(0.93-6.08)$ \\
AC & 53 & 49 & 1.74 & $(0.99-2.29)$ \\
AA & 21 & 13 & 1.51 & \\
AA+AC & 74 & 62 & 1.00 &
\end{tabular}

hOGG1, human homolog of the 8-oxoguanine DNA glycosylase 1; ERCC2/XPD, excision repair cross-complementing rodent repair deficiency complementation group 2/Xeroderma pigmentosum complementation group D; PCa, prostate cancer; OR, odds ratio; CI, confidence interval.

There was no significant difference in mean age between cases and controls (Table I). The Chi-square ${ }_{\mathrm{HWE}}$ for genotype distributions was $>0.05$ among controls. The genotype frequencies among controls did not deviate from the expected distributions based on the HWE $\left(\mathrm{P}_{\mathrm{XPD}}=0.65\right.$ vs. $\left.\mathrm{P}_{\mathrm{hOGG}}=0.096\right)$

Association of XPD Arg156Arg and hOGG1 Ser326Cys polymorphisms with $P C a$. There was no significant association between the genotypes or alleles of the two polymorphisms and the occurrence of PCa (Table II). A previous study by Zheng et al (15) demonstrated that each single-nucleotide polymorphism (SNP) is only moderately associated with $\mathrm{PCa}$; however, when SNPs are combined, the association may be more significant. The combined effect of XPD Arg156Arg and hOGG1 Ser326Cys was also investigated and no statistically significant association was observed.

To evaluate the role of these polymorphisms with regard to susceptibility to $\mathrm{PCa}, \mathrm{PCa}$ patients were stratified according to risk levels. The distribution of genotypes and frequency of alleles among the groups of patients with different risk levels is shown in Table III.

According to the European Association of Urology (EAU) guidelines for prostate cancer (16), patients were divided into three groups: i) low-risk (localised PCa: cT1-T2a or Gleason score 2-6 or PSA $<10$ ); ii) intermediate-risk (localised PCa: cT2b-T2c or Gleason score 7 or PSA 10-20); and iii) high-risk (localised PCa: $\geq$ cT3a or Gleason score 8-10 or PSA $>20$ ).

For the XPD156 $\mathrm{C} \rightarrow \mathrm{A}$ polymorphism, the frequency of the XPD156 AA genotype in high-risk PCa patients was significantly higher in comparison to controls $(\mathrm{OR}=3.80$; 95\% CI: 1.19-12.18; $\mathrm{P}=0.017$ ), conferring an $\sim 3$-fold increase in the risk for cancer. Individuals with the heterozygous $\mathrm{AC}$ genotype were associated with increased risk of $\mathrm{PCa}$ $(\mathrm{OR}=2.48 ; 95 \% \mathrm{CI}: 1.02-6.35 ; \mathrm{P}=0.033)$. In addition, significant differences were observed $(\mathrm{OR}=2.76 ; 95 \% \mathrm{CI}$ : 1.18-6.84;

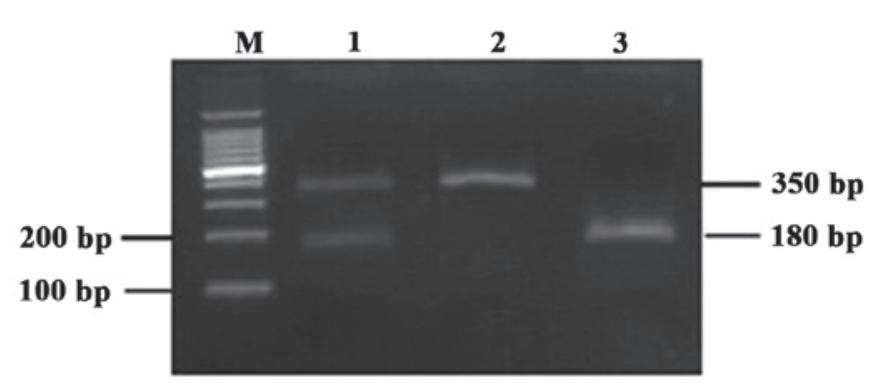

Figure 1. Representative polymerase chain reaction-restriction fragment length polymorphism analysis of the Xeroderma pigmentosum complementation group D Arg156Arg polymorphism. Lane M, DNA marker; lane 1, the AC heterozygote contains the 350- and 180-bp bands following restriction digestion; lane 2, the $\mathrm{CC}$ homozygote is not cleaved by Tfi and remains a single 350 -bp band; lane 3, the AA homozygote is cleaved by $T f i$ and yields overlapping 180- and 170-bp bands.

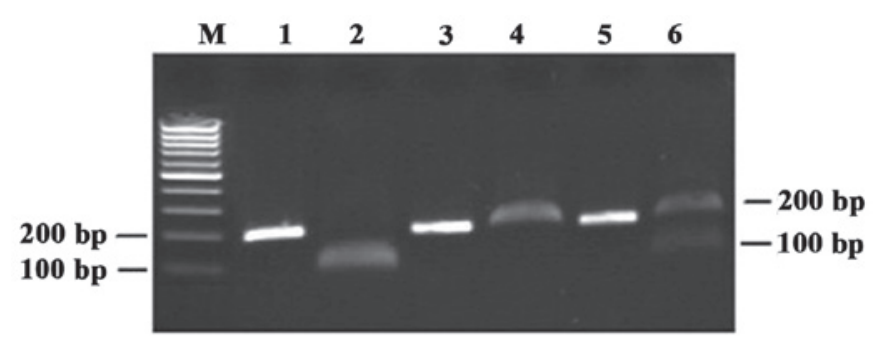

Figure 2. Representative polymerase chain reaction-restriction fragment length polymorphism analysis of the human homolog of the 8-oxoguanine DNA glycosylase 1 Ser326Cys polymorphism. Lane M, DNA marker; lanes 1,3 and 5, the PCR product prior to digestion was a 200-bp band; lane 2, the Cys/Cys homozygote is cleaved by Fnu4HI and yields a 100-bp band; lane 4, the Ser/Ser homozygote is not cleaved by Fnu4HI and remains a single 200-bp band; lane 6, the Ser/Cys heterozygote contains the 200- and 100-bp bands following restriction digestion. 
Table III. Association of Ser326Cys polymorphism of the hOGG1 gene and Arg156Arg polymorphism of the ERCC2/XPD gene with PCa aggressiveness.

\begin{tabular}{|c|c|c|c|c|c|c|c|c|c|c|c|c|}
\hline $\begin{array}{l}\text { Polymorphism } \\
\text { genotype or allele }\end{array}$ & HR & OR & $95 \%$ CI & $\mathrm{P}$-value & MR & OR & $95 \%$ CI & $\mathrm{P}$-value & LR & OR & $95 \%$ CI & P-value \\
\hline \multicolumn{13}{|l|}{ Arg156Arg } \\
\hline $\mathrm{AA}$ & 13 & 3.80 & $1.19-12.18$ & 0.017 & 7 & 1.86 & $0.50-6.60$ & 0.366 & 1 & 0.58 & $0.01-6.02$ & 1.000 \\
\hline $\mathrm{AC}$ & 32 & 2.48 & $1.02-6.35$ & 0.033 & 13 & 0.92 & $0.34-2.54$ & 1.000 & 8 & 1.24 & $0.33-5.22$ & 0.773 \\
\hline $\mathrm{CC}$ & 10 & 1.00 & & & 11 & 1.00 & & & 5 & 1.00 & & \\
\hline $\mathrm{AA}+\mathrm{AC}$ & 45 & 2.76 & $1.18-6.84$ & 0.011 & 20 & 1.11 & $0.45-2.87$ & 0.835 & 9 & 1.10 & $0.30-4.51$ & 1.000 \\
\hline $\mathrm{A}$ & 58 & 1.86 & $1.13-3.06$ & 0.012 & 27 & 1.29 & $0.69-2.38$ & 0.456 & 10 & 0.93 & $0.36-2.25$ & 1.000 \\
\hline $\mathrm{C}$ & 52 & 1.00 & & & 35 & 1.00 & & & 18 & 1.00 & & \\
\hline \multicolumn{13}{|l|}{ Ser326Cys } \\
\hline GG & 17 & 2.93 & $1.00-8.74$ & 0.033 & 6 & 1.78 & $0.41-7.48$ & 0.510 & 3 & 2.07 & $0.24-17.26$ & 0.406 \\
\hline $\mathrm{CG}$ & 26 & 1.10 & $0.47-2.76$ & 1 & 18 & 1.31 & $0.45-4.13$ & 0.637 & 8 & 1.36 & $0.30-8.51$ & 1.000 \\
\hline $\mathrm{CC}$ & 12 & 1.00 & & & 7 & 1.00 & & & 3 & 1.00 & & \\
\hline $\mathrm{GG}+\mathrm{CG}$ & 43 & 1.46 & $0.64-3.49$ & 0.447 & 24 & 1.40 & $0.51-4.27$ & 0.646 & 11 & 1.46 & $0.35-8.69$ & 0.754 \\
\hline $\mathrm{G}$ & 60 & 1.62 & $1.00-2.67$ & 0.044 & 30 & 1.27 & $0.69-2.34$ & 0.465 & 14 & 1.35 & $0.56-3.24$ & 0.543 \\
\hline $\mathrm{C}$ & 50 & 1.00 & & & 32 & 1.00 & & & 14 & 1.00 & & \\
\hline
\end{tabular}

hOGG1, human homolog of the 8-oxoguanine DNA glycosylase 1; ERCC2/XPD, excision repair cross-complementing rodent repair deficiency complementation group 2/Xeroderma pigmentosum complementation group D; PCa, prostate cancer; OR, odds ratio; CI, confidence interval; HR, high-risk; MR, intermediate-risk; LR, low-risk.

$\mathrm{P}=0.011)$ when variant-containing genotypes were combined (AC+AA) and compared to the homozygous wild-type (CC). The frequency of the XPD156 A allele was 0.527 among the high-risk cases and 0.375 among the controls, a difference described as statistically significant (Chi-square $\mathrm{P}=0.012$ ).

For Ser326 C $\rightarrow \mathrm{G}$, the high-risk individuals with the homozygous GG genotype exhibited a significantly increased risk of $\mathrm{PCa}(\mathrm{OR}=2.93 ; 95 \% \mathrm{CI}: 1-8.74 ; \mathrm{P}=0.033)$, which reduced the rate of DNA repair compared to the CC or GC genotypes. However, no significant difference was observed $(\mathrm{OR}=1.46$; 95\% CI: 0.64-3.49; $\mathrm{P}=0.447)$ when variant-containing genotypes were combined (GC+GG). The frequency of the Ser326 $\mathrm{G}$ allele was 0.545 among the high-risk cases and 0.425 among the controls, a difference described as statistically significant (Chi-square $\mathrm{P}=0.044)$.

For the low- and intermediate-risk groups, no differences were observed in the distribution of the genotypes of either polymorphism between the two groups (Table III).

\section{Discussion}

In this population-based study, the association between the XPD and hOGG1 gene polymorphisms and PCa risk was investigated in 100 cases and 100 controls. No association was observed between any of these SNPs and the overall risk of $\mathrm{PCa}$. As regards the clinical characteristics of $\mathrm{PCa}$, the roles of the Arg156Arg polymorphism of the ERCC2/XPD gene and the Ser326Cys polymorphism of the hOGG1 gene in PCa aggressiveness were assessed. Significant differences were identified between the two DNA repair genes and the high-risk level group (Table III).

As regards ERCC2/XPD SNPs, several studies investigating the possible association between ERCC2 variants in DNA repair genes and cancer risk have been published $(17,18)$. The most extensively investigated ERCC2 polymorphisms were Asp312Asn and Lys751Gln. ERCC2 Arg156Arg is a silent C/A polymorphism and thus it may exert an effect only at the transcriptional level, if ERCC2 Arg156Arg is the biologically effective polymorphism. The reason for a higher expression level of ERCC2 being associated with an increased cancer risk remains to be elucidated. According to a previous study by Vogel et al (19), several genes in that region, including the apoptosis-controlling gene RAI, ERCC1 and ERCC2/XPD, correlate strongly with each other in their expression levels. Therefore, an accompanying higher expression of another gene may confer increased cancer risk. Previous studies demonstrated that XPD codon 156 polymorphisms were associated with lung (20), bladder (21) and breast cancer (22) in Chinese populations. To the best of our knowledge, the present study was the first to demonstrate that the silent polymorphisms of XPD156 affect the risk of PCa. Our main finding was that the A allele of XPD Arg156Arg is associated with predisposition to high-risk PCa (AA: OR=3.80; 95\% CI: 1.19-12.18; $\mathrm{P}=0.017$; $\mathrm{AC}: \mathrm{OR}=2.48 ; 95 \%$ CI: $1.02-6.35 ; \mathrm{P}=0.033$; $\mathrm{A}+\mathrm{AC}$ : $\mathrm{OR}=2.76 ; 95 \% \mathrm{CI}: 1.18-6.84 ; \mathrm{P}=0.011 ;$ and $\mathrm{A}: \mathrm{OR}=1.86$; 95\% CI: $1.13-3.06 ; \mathrm{P}=0.012$ ).

Previous epidemiological studies associated the Ser326Cys polymorphism in the hOGG1 gene with the risk of various types of cancer $(23,24)$. Significant risk increases were reported in subjects with the hOGG1 326 polymorphism for these cancers. In a study by Srivastava et al (25), the homozygous variant genotypes of hOGG1 Ser326Cys polymorphisms exhibited a statistically significant increased risk for gallbladder carcinogenesis $(\mathrm{OR}=2.5$; 95\% CI: 1.1-5.4; $\mathrm{P}=026)$. The risk for variant-containing genotypes $(\mathrm{CG}+\mathrm{GG})$ of hOGG1 Ser326Cys was also significant (OR=1.8; 95\% CI: 1.2-2.6; $\mathrm{P}=002$ ) when compared to the homozygous wild-type CC genotype. A previous study by Arizono et al (26) indicated that the frequency of the hOGG1 codon 326 GG genotype was significantly higher in bladder cancer cases compared to the 
controls. The homozygous GG genotype exhibited a significant association with lung cancer compared to the $\mathrm{C}$ allele carrier status in previous studies $(27,28)$. In the present study, individuals who were homozygous for the GG genotype were at a markedly increased risk for developing $\mathrm{PCa}(\mathrm{OR}=2.93$; 95\% CI: 1-8.74; $\mathrm{P}=0.033$ ).

Although Ser326Cys polymorphisms have been extensively investigated in $\mathrm{PCa}$, the conclusions are contradictory. $\mathrm{Xu}$ et al (29) observed that men with the CC genotype (Ser326) had an increased risk of PCa, particularly the sporadic form. Zhang et al (30) demostrated that, following adjustment for confounders, subjects who were heterozygous or homozygous for the variant allele of the hOGG1 Ser326Cys polymorphism appeared to experience a lower risk of PCa compared to those who were homozygous for the wild-type allele $(\mathrm{OR}=0.72$ and 95\% CI: 0.46-1.10). Nam et al (31) investigated a panel of 13 polymorphisms in 13 different genes in patients with the GG and the CC genotype of the hOGG1-326 gene (OR=0.68; 95\% CI: 0.5-1.0; $\mathrm{P}=0.05$ ). By contrast, Chen et al (32) demonstrated that a significantly increased PCa risk was observed in subjects with at least one hOGG1326Cys allele $\left(\mathrm{OR}_{\mathrm{adj}}=2.1\right.$; 95\% CI: 1.2-3.8). These significant risk changes were observed for subjects with the heterozygous hOGG1 326Ser/Cys genotype $\left(\mathrm{OR}_{\mathrm{adj}}=1.8 ; 95 \% \mathrm{CI}: 1.01-3.3\right)$ and with the homozygous hOGG1 326Cys/Cys genotype $\left(\mathrm{OR}_{\mathrm{adj}}=7.8\right.$; $\left.95 \% \mathrm{CI}: 1.7-36.2\right)$. To the best of our knowledge, our study is the first to provide evidence that men bearing the GG genotype (Cys326) are susceptible to the development of high-risk PCa. Our results may encourage studies on larger populations. Xu et al (29) also indicated that Cys326 confers increased cancer risk in Asian populations, a finding that was similar to ours.

Our study has certain limitations. First, increasing the number of individuals may increase the statistical power of the study. Second, the follow-up study may provide more precise results, as we were not able to ensure a lifelong cancer-free status in the controls.

In conclusion, the findings of our study suggest that the ERCC2/XPD Arg156Arg and hOGG1 Ser326Cys polymorphisms contribute to high-risk PCa susceptibility in a Chinese population.

\section{References}

1. Jemal A, Siegel R, Ward E, Hao Y, Xu J, Murray T and Thun MJ: Cancer statistics, 2008. CA Cancer J Clin 58: 71-96, 2008.

2. McCracken M, Olsen M, Chen MS Jr, Jemal A, Thun M, Cokkinides V, Deapen D and Ward E: Cancer incidence, mortality, and associated risk factors among Asian Americans of Chinese, Filipino, Vietnamese, Korean, and Japanese ethnicities. CA Cancer J Clin 57: 190-205, 2007.

3. Pienta KJ and Esper PS: Risk factors for prostate cancer. Ann Intern Med 118: 793-803, 1993.

4. Chan JM, Holick CN, Leitzmann MF, et al: Diet after diagnosis and the risk of prostate cancer progression, recurrence, and death (United States). Cancer Causes Control 17: 199-208, 2006.

5. Vogel U, Olsen A, Wallin H, Overvad K, Tjonneland A and Nexo BA: Effect of polymorphisms in XPD, RAI, ASE-1 and ERCC1 on the risk of basal cell carcinoma among Caucasians after age 50. Cancer Detect Prev 29: 209-214, 2005.

6. Thompson LH and West MG: XRCC1 keeps DNA from getting stranded. Mutat Res 459: 1-18, 2000.

7. Sancar A and Tang MS: Nucleotide excision repair. Photochem Photobiol 57: 905-921, 1993.

8. Ishikawa T, Zhang SS, Qin X, et al: DNA repair and cancer: lessons from mutant mouse models. Cancer Sci 95: 112-117, 1993.
9. Terry MB, Gammon MD, Zhang FF, Eng SM, Sagiv SK, Paykin AB, Wang Q, Hayes S, Teitelbaum SL, Neugut AI and Santella RM: Polymorphism in the DNA repair gene XPD, polycyclic aromatic hydrocarbon-DNA adducts, cigarette smoking, and breast cancer risk. Cancer Epidemiol Biomarkers Prev 13: 2053-2058, 2004.

10. Goode EL, Ulrich CM and Potter JD: Polymorphisms in DNA repair gene and associations with cancer risk. Cancer Epidemiol Biomarkers Prev 11: 1513-1530, 2002.

11. Xing D, Tan W and Lin D: Genetic polymorphisms and susceptibility to esophageal cancer among Chinese population (Review). Oncol Rep 10: 1615-1623, 2003.

12. Park J, Chen L, Tockman MS, Elahi A and Lazarus P: The human 8-oxoguanine DNA N-glycosylase 1 (hOGG1) DNA repair enzyme and its association with lung cancer risk. Pharmacogenetics 14: 103-109, 2004.

13. Kim JI, Park YJ, Kim KH, Kim JI, Song BJ, Lee MS, Kim CN and Chang SH: hOGG1 Ser326Cys polymorphism modifies the significance of the environmental risk factor for colon cancer. World J Gastroenterol 9: 956-960, 2003.

14. Song FJ, Zhang BL, He M, et al: Trend analysis of the incidence of prostate cancer in Tianjin between 1981 and 2004. Zhong hua Yi Xue Za Zhi 90: 2811-2814, 2010 (In Chinese).

15. Zheng SL, Sun J, Wiklund F, et al: Cumulative association of five genetic variants with prostate cancer. N Engl J Med 358: 910-919, 2008.

16. Heidenreich A, Aus G, Bolla M, et al: Guidelines on Prostate Cancer. European Association of Urology (EAU), Arnhem, The Netherlands, 2010.

17. Yuan H, Niu YM, Wang RX, Li HZ and Chen N: Association between XPD Lys751Gln polymorphism and risk of head and neck cancer: a meta-analysis. Genet Mol Res 22: 3356-3364, 2011.

18. Ding DP, Ma WL, He XF and Zhang Y: XPD Lys751Gln polymorphism and esophageal cancer susceptibility: a meta-analysis of case-control studies. Mol Biol Rep 39: 2533-2540, 2012.

19. Vogel U, Nexo BA, Tjonneland A, et al: ERCC1, XPD and RAI mRNA levels in lymphocytes are not associated with lung cancer risk in a prospective study of Danes. Mutat Res 593: 88-96, 2006.

20. Wu W, Zhang W, Qiao R, et al: Association of XPD polymorphisms with severe toxicity in non-small cell lung cancer patients in a Chinese population. Clin Cancer Res 15: 3889-3895, 2009.

21. Shao J, Gu M, Xu Z, et al: Polymorphisms of the DNA gene XPD and risk of bladder cancer in a Southeastern Chinese population. Cancer Genet Cytogenet 177: 30-36, 2007.

22. Yin J, Liang D, Vogel U, et al: The polymorphism of DNA repair gene ERCC2/XPD Arg156Arg and susceptibility to breast cancer in a Chinese population. Biochem Genet 47: 582-590, 2009.

23. Guan P, Huang D, Yin Z and Zhou B: Association of the hOGG1 Ser326Cys polymorphism with increased lung cancer susceptibility in Asians: a meta-analysis of 18 studies including 7592 cases and 8129 controls. Asian Pac J Cancer Prev 12: 1067-1072. 2011.

24. Ni M, Qiu J, He W and Wang X: The functional Ser326Cys polymorphism in hOGG1 is associated with gastric cancer risk: evidence from 1180 cases and 2444 controls.Eur J Gastroenterol Hepatol 24: 683-687, 2012.

25. Srivastava K, Srivastava A and Mittal B: Polymorphisms in ERCC2, MSH2, and OGG1 DNA repair genes and gallbladder cancer risk in a population of Northern India. Cancer 116: 3160-3169, 2010.

26. Arizono K, Osada Y and Kuroda Y: DNA repair gene hOGG1 codon 326 and XRCC1 codon 399 polymorphisms and bladder cancer risk in a Japanese population. Jpn J Clin Oncol 38: 186-191, 2008.

27. Okasaka T, Matsuo K, Suzuki T, et al: hOGG1 Ser326Cys polymorphism and risk of lung cancer by histological type. J Hum Genet 54: 739-745, 2009.

28. Li H, Hao X and Zhang W: The hOGG1 Ser326Cys polymorphism and lung cancer risk: a meta-analysis. Cancer Epidemiol Biomarkers Prev 17: 1739-1745, 2008.

29. Xu J, Zheng SL, Turner A, et al: Associations between hOGG1 sequence variants and prostate cancer susceptibility. Cancer Res 62: 2253-2257, 2002.

30. Zhang J, Dhakal IB, Greene G, Lang NP and Kadlubar FF: Polymorphisms in hOGG1 and XRCC1 and risk of prostate cancer: effects modified by plasma antioxidants. Urology 75: 779-785, 2010.

31. Nam RK, Zhang WW, Jewett MA, et al: The use of genetic markers to determine risk for prostate cancer at prostate biopsy. Clin Cancer Res 11: 8391-8397, 2005.

32. Chen L, Elahi A, Pow-Sang J, Lazarus P and Park J: Association between polymorphism of human oxoguanine glycosylase 1 and risk of prostate cancer. J Urol 170 (6 Pt 1): 2471-2474, 2003. 